\title{
La renovación de los métodos de enseñanza de la lectura en la España de finales del siglo XVIII y comienzos del XIX: la figura de Vicente Naharro
}

\section{The Renewal of Teaching Methods for Reading in the Late $18^{\text {th }}$ and Early $19^{\text {th }}$ Century Spain: the Figure of Vicente Naharro}

\author{
Vicente CALVO FERNÁNDEZ \\ Universidad Rey Juan Carlos
}

Recibido: Mayo 2012

Aceptado: Septiembre 2012

\section{Resumen}

En este trabajo, se investiga la contribución de Vicente Naharro a la renovación de los métodos de lectura que se empleaban en la escuela elemental en la España de finales del XVIII y comienzos del XIX. Este maestro aragonés afincado en Madrid aplicó en las escuelas que regentó un método de lectoescritura que él mismo calificaba de racional y orgánico y que, en gran medida, supone una ruptura con los usos pedagógicos vigentes en la época.

Palabras clave: Lectoescritura, siglo XIX, Historia de la Educación, Escuela Elemental, Vicente Naharro, Métodos de Enseñanza.

\begin{abstract}
What is examined in this paper is the contribution of Vicente Naharro to the renewal of reading methods, which were used in elementary schools in Spain in the late eighteenth and early nineteenth centuries. This teacher applied a literacy method in schools, which he described as rational and organic and, to a large extent, represents a radical overhaul of the traditional educational uses of the time.
\end{abstract}

Keywords: Literacy, Nineteenth Century, History of Education, Elementary School, Vicente Naharro, teaching Methods.

Nos proponemos, en este trabajo, investigar la contribución de Vicente Naharro a la renovación de los métodos de lectura que se empleaban en la escuela elemental en la España de finales del XVIII y comienzos del XIX.

Estas coordenadas cronológicas obedecen al hecho de que es en esta época cuando se establecen las bases racionales para la organización escolar, comienza a darse una reflexión más general, honda y sistemática acerca de los métodos de enseñanza y, en 
consecuencia, empiezan a editarse libros de texto escolares que responden a un plan pedagógico meditado y bien definido.

Puesto que el corpus de textos es amplio desde el último tercio del siglo XVIII, limitaremos nuestro trabajo -como decimos- a la obra de Vicente Naharro, defensor de un método sintético-silábico que él mismo califica de "racional" y "orgánico".

Existen varios e importantes estudios de carácter general sobre la enseñanza de la lectura en la época que nos ocupa. Vale la pena, en este sentido, destacar el de García Hurtado (2004), que trata de las cartillas de lectura entre 1700 y 1780 y, sobre todo, son fundamentales los trabajos de Viñao $(1997,2001,2002)$ y los de Ruiz Berrio (1997, 2004, entre otros), que se centran en la situación de la educación al final del Antiguo Régimen y en la evolución de los métodos de enseñanza a lo largo del XIX.

Las fuentes primarias, hasta el XVIII inclusive, fueron felizmente recopiladas por Infantes (1998) e Infantes y Martínez Pereira (2003). Sin embargo, a pesar de que contamos con este extenso e interesante material, hay aún poca literatura científica y falta un trabajo de investigación pormenorizado sobre autores concretos. La búsqueda de fuentes secundarias en repertorios bibliográficos como BiTe (cf. Esparza et al., 2010) solo sirve para corroborar que estamos ante un terreno relativamente poco cultivado, en el que aún no se han publicado muchos trabajos específicos ${ }^{1}$.

Además, está por establecer el corpus del siglo XIX, pero el número de referencias en bases de datos bibliográficas como BICRES (cf. Esparza y Niederehe et al., 2012) hace pensar que el tema merece atención, pues hallamos una cantidad significativa de textos diferentes, en torno a una veintena (solo entre 1801 y 1860 sin tener en cuenta reediciones), de los que no existen, hasta donde conocemos, ni ediciones ni estudios recientes.

\section{La polémica del método}

Para poder contextualizar nuestro objeto de estudio, conviene siquiera un breve excurso acerca de los métodos de lectoescritura.

Uno de los debates más candentes entre los especialistas en educación elemental ha sido siempre la elección del método para la enseñanza de la lectura y escritura. Desde hace décadas hay una extensísima literatura sobre cuál es el más adecuado y existen casi tantas propuestas como maestros. Por ello, y porque hasta la más sucinta exposición del tema excedería por completo los límites de este trabajo, ofreceremos ahora un esquema que resume los principales enfoques metodológicos, aun conscientes de que cualquier intento taxonómico resulta insuficiente. Se pretende, por tanto, situar el objeto de nuestra investigación en unos parámetros epistemológicos siguiendo la nomenclatura acuñada por la tradición científica (cf. por ejemplo Lebrero y Lebrero, $\left.{ }^{4} 1999,47 \mathrm{ss}\right)$.

${ }^{1}$ Uno de los pocos ejemplos de estudios sobre autores concretos es el de Calero Vaquera (2007), que se interesó por la obra de Juan Robles, maestro del XVII. 
Los métodos tradicionales ${ }^{2}$ para el aprendizaje de la lectura suelen clasificarse, según el punto de partida y el sentido que adopta dicho aprendizaje, como sigue:

a) Si se parte de unidades menores, distintivas, no significativas, en las que se descompone el discurso oral o escrito, hasta llegar a unidades significativas, se habla de métodos sintéticos (o bottom-up).

b) $\mathrm{Si}$, por el contrario, lo primero que se presenta al discente son unidades mayores, dotadas de significado, y se desciende, a través de la explicación de sus componentes, hasta las unidades menores (sílabas o letras/sonidos), los métodos se denominan analíticos (o top-down).

Estas dos posibilidades obedecen a la propia estructura de las lenguas de nuestro entorno cultural, que se caracterizan por estar constituidas por signos de doble articulación, según la afortunada expresión de Martinet. Pero también son reveladores de una determinada concepción sobre la psicología humana y los procesos de percepción y aprendizaje:

-Los métodos sintéticos permiten una rápida adquisición del mecanismo de desciframiento y que el discente logre ser un lector autónomo, además de facilitar desde el principio la articulación correcta. Por eso, se utilizan frecuentemente en reeducación y en la instrucción de sujetos con necesidades educativas especiales.

-Los métodos analíticos son más lentos, pero responden a la idea de que la percepción humana es global (Teoría de la Gestalt) y a la propia fisiología de la lectura, que se produce mediante movimientos oculares amplios y discontinuos (sacádicos). Además, los métodos analíticos fomentan la motivación y el llamado aprendizaje significativo, ya que el discente puede comenzar manejando unidades con significado que remiten a referentes de su universo de intereses, y no a simples asociaciones (por ejemplo, un grafema $=$ un sonido) que, aisladamente o en primer término, aparezcan sin sentido.

Por otro lado, no son indiferentes las características peculiares del código. Hay lenguas como francés, en las que existe un grado menor de correspondencia entre sus sistemas gráfico y fónico que, por ejemplo, en español o italiano. En aquellos casos (menos correspondencia) puede resultar más arduo para el discente el aprendizaje mediante un método sintético porque, evidentemente, se multiplican las reglas de desciframiento. No en vano, a lo largo de la historia la nómina de defensores de los métodos analíticos está constituida en gran parte por autores francófonos (Randovilliers, Itard, Seguin, Jacotot, Decroly, por citar algunos).

Los enfoques, dentro de estas dos grandes líneas, han sido múltiples. Dentro de los métodos sintéticos se ha enseñado la lectura partiendo de los nombres de las letras (deletreo), o bien de los sonidos aislados, o bien mediante las sílabas. Los métodos analíticos también se han aplicado de modos muy distintos: sin intervención del

\footnotetext{
${ }^{2}$ Usamos, de intento, la etiqueta "tradicional" para distinguir estos métodos de lectoescritura de las actuales perspectivas constructivistas.
} 
educador, demorando más o menos el proceso de descomposición. De hecho, una gran parte de los métodos han terminado siendo mixtos para fomentar desde el principio, tanto el aprendizaje de las reglas de desciframiento como el descubrimiento global del significado.

Una vez presentado el marco, vamos a ubicar en estos parámetros los textos para la enseñanza de la lectura en la España del siglo de las luces.

\section{La enseñanza de la lectura en la España de finales del XVIII}

Los expertos en la historiografía de la educación en España coinciden en señalar el periodo ilustrado como la época en la que se asentaron las bases de todas las reformas posteriores que afectaron al modo de enseñar la lectura. Ruiz Berrio $(2004,132)$ apunta, concretamente, a la creación en 1788 de la Escuela de la Real Comitiva (posteriormente de San Isidro), en lo que sería el primer hito en la renovación de los estudios elementales. Podría considerarse que dicho establecimiento de San Isidro fue, avant la lettre, el embrión de los centros de Magisterio en España y el conjunto de escuelas reales que siguieron a su fundación constituye lo que se ha dado en llamar "movimiento de San Ildefonso". En el seno de este movimiento reformista cambia el enfoque de la enseñanza tradicional de la lectura y empieza a abandonarse el deletreo.

Efectivamente, desde la Antigüedad venía enseñándose a leer por el método alfabético: la eme con la a, "ma". Después se pasaba a las sílabas, que también se deletreaban, y finalmente a las palabras. Afirma Viñao (2002a, 349):

Las conocidas cartillas impresas — "beceroles" en Cataluña - en las que se aprendía a leer en las escuelas de la Europa del Antiguo Régimen, desde el siglo XVI al XVIII, se ordenaban en función de este método (incluso las primeras de ellas estaban escritas en latín, no en la lengua materna). Su contenido usual era, más o menos por este orden, el siguiente: el abecedario, un silabario, las oraciones más comunes, un catecismo elemental, el orden para ayudar a Misa —en los países católicos - y una tabla de multiplicar. Todo ello en 16 páginas y tamaño $8^{\circ}$. Este era el texto oficial, por así decirlo, junto al que otras veces — sobre todo cuando ya se leía de corrido - se recurría a textos manuscritos - procesos, documentos - o impresos, desde los también bendecidos catones a los denostados pliegos sueltos.

Algunos de los maestros ilustrados abominaban de este sistema y empezaron a proponer nuevas formas de enseñanza, entre las que se incluyen tres propuestas:

a) La sustitución del método alfabético tradicional por el silábico-fonético.

b) El aprendizaje simultáneo de la escritura.

c) La introducción de material "didáctico": juegos, artilugios, ilustraciones (cf. Viñao, 2002a, 350), que empiezan a utilizar, sobre todo, los escolapios.

Entre estos maestros reformistas se encuentra don Vicente Naharro, cuya contribución a la historia de la enseñanza de la lectura y escritura pasamos a analizar a continuación. 


\section{La contribución de Naharro}

Vicente Naharro (1750-1823) fue un maestro aragonés muy ligado al movimiento ilustrado. Según el Diccionario biográfico y bibliográfico de calígrafos españoles, de Cotarelo y Mori (cf. 1916, 100), nació en Tobed, cerca de Calatayud, en 1750, y murió en Madrid, el año 1823. Estudió Filosofía y Letras en la Universidad de Zaragoza, desempeñó los cargos de vicerrector y rector del Colegio de Santo Tomás y llegó a ser nombrado "Maestro Real y del Número" de la Corte y "Examinador de los de su Arte y Revisor de firmas y letras sospechosas por el Supremo Consejo", tal como figura en la portada de algunas de sus obras.

Cotarelo afirma $(1916,100)$ que la primera escuela la abrió en Madrid el año 1782, aunque es el propio maestro quien asegura, en el prólogo de la segunda edición de su Nuevo Arte (cf. 1824, 15), que fue a partir de 1786 cuando comenzó a aplicar en sus clases los métodos de lectura y escritura que él mismo había ido diseñando.

En 1791 se crearon en Madrid ocho Escuelas Reales, una en cada cuartel, con sueldo de 400 ducados, casa y pasante, para recompensar a los ocho maestros que más se habían distinguido en la enseñanza del Método de Anduaga, y Naharro fue nombrado rector de una de ellas (cf. Cotarelo, 1916, 101). Precisamente para uso en esta escuela publicó la primera versión de su Silabario, como diremos más adelante, que fue rechazado por la Academia ${ }^{3}$. Naharro tuvo ocasión de resarcirse de este contratiempo, pues, al final de su vida, la Junta de Enseñanza, que había convocado un concurso público, le otorgó en 1817 un premio de 2.000 reales por elaborar el mejor método para la educación de la infancia. De esto, por cierto, se congratula su yerno y heredero en la presentación, ya póstuma, del Nuevo Arte (cf. 1824, 13).

No resulta sencillo elaborar un elenco de las obras de Naharro por varios motivos: primero, porque no han aparecido exentas algunas de las que él mismo nombra, bien porque no las llegó a dar a la imprenta y solo figuran manuscritas, bien porque parte de su producción se encuentra aneja a la obra de otros autores. Además, Naharro reconoce que varias veces fue incorporando mejoras a sus métodos. En cualquier caso, hay múltiples ediciones y reediciones hasta entrado el siglo XX, tanto en España como en América, lo cual habla bien del grado de aceptación con que fueron recibidos sus métodos en las escuelas de primera enseñanza. La lista de obras que el autor reconoce es la que sigue ${ }^{4}$.

- Métodos de lectura:

${ }^{3}$ Este veto de la Academia no fue el único que sufrió Naharro en su vida, ya que en 1818 la censura retuvo otra de sus obras principales, El Arte de enseñar a escribir cursivo y liberal, que no pudo editarse hasta 1820 .

${ }^{4}$ Distinguimos, de un modo un tanto artificial, los textos destinados a los niños, de las obras en las que Naharro justifica su método, obviamente orientadas a maestros y educadores. 
-1787: [Silabario].

Cotarelo $(1916,102)$ data la obra en este año dando fe a las palabras de su autor. Infantes y Martínez Pereira $(2003,97)$ no localizan ni esta edición, ni su reedición un año después, de la que Ruiz Berrio sí da noticia. Tampoco Niederehe (2005), cuya fuente es Aguilar Piñal, puede determinar la fecha exacta.

-1791: Cartilla o Silabario para uso de las Reales Escuelas del Sitio de San Ildefonso de la comitiva de S. M. y de San Isidro de esta Corte.

Está incluido, según palabras del autor, en la obra de José de Anduaga y Garimberti Tratado sobre el modo de enseñar el conocimiento de las letras, y su unión en sílabas y en dicciones, para uso de las Reales Escuelas del Sitio de San Ildefonso de la comitiva de S. M. y de San Isidro de esta Corte. En la Imprenta Real. Año de 1791, cuya portada reproducen Infantes y Martínez Pereira $(2003,829)$, quienes transcriben a continuación la cartilla completa.

-s.f.: Libro segundo de los niños. Madrid, Imprenta. Real.

La cita Cotarelo $(1916,102)$ a tenor de las palabras de Naharro en el prólogo del Nuevo Arte.

\section{-1802: Silabario ó Método práctico de enseñar á leer}

La fecha la ofrece Naharro cuando afirma en el prólogo del Nuevo Arte que, anejo a la Recopilación, incluyó su propio silabario y el método de enseñar a leer (la doble denominación, en realidad, corresponde a una misma obra, tal como reza el título). Viñao $(1998,262)$ halló la primera versión del texto al final del manuscrito de la Recopilación, en el Archivo Histórico Nacional, Consejos, leg. 5 549-31, f. 284-298. En cualquier caso, el Método práctico se edita exento desde esta época más o menos hasta principios del siglo XX. Cotarelo afirma de la obra $(1916,104)$ : "La que poseo, y es la más antigua que he visto, lleva este título: Método practico de enseñar á leer por Don Vicente Naharro, Maestro Real, y del Colegio de esta corte. Revisor de firmas por el Supremo Consejo, etc. Sexta edición. Madrid, MDCCCXV. Imprenta de Núñez. Con licencia. 8. ${ }^{\circ}$; 96 págs. El ejemplar de edición más antigua de la Biblioteca Nacional lleva esta portada: Método práctico de enseñar á leer, por Don Vicente Naharro, Profesor de educación de esta corte, Revisor de firmas por el Gobierno, etc. Octava edición. Madrid, Imprenta que fué de Fuentenebro, 1821. 8. ${ }^{\circ} ; 96$ págs.".

-1815: Silabario para el Nuevo Arte de enseñar a leer a los niños de las Escuelas por el orden de un Alfabeto Racional. Por D. Vicente Naharro, Maestro Real, y del Colegio de esta corte, revisor de firmas por el Supremo Consejo, etc. Madrid MDCCCXV. Imprenta de Núñez.

-s.f.: Reglas instructivas de la Ortografia. 
El autor la cita entre sus obras al final de su Descripción de los juegos de la infancia (cf. Cotarelo, 1916, 105), pero no se ha encontrado texto alguno, ni impreso ni manuscrito.

- Ensayos sobre temas didácticos, dirigidos a maestros

-1802: Recopilación de los varios métodos inventados para facilitar la enseñanza de leer. Por D. Vicente Naharro, Maestro Real y del Número de esta corte, Examinador de los de su [iarte?] Revisor de firmas y letras sospechosas, etc. Madrid, En la oficina de $D[\ldots, 1802]$.

Cotarelo lo data basándose en la información que da el propio autor en el prólogo del Nuevo Arte, pero la fecha y el impresor no figuran, tal vez porque el ejemplar de la Biblioteca Nacional (signatura 2/30136) tiene la portada rasgada.

-1814: Nueva Arte de enseñar á leer á los niños de las escuelas, dispuesto por el orden de un alfabeto racional. Madrid.

Tampoco ha aparecido esta primera edición, de la que habla el autor en el Método práctico de 1815 al que se refiere Cotarelo $(1916,103)$. En este trabajo nos basamos en la edición más antigua que hemos encontrado, titulada: $E l$ Nuevo Arte de enseñar a leer a los niños de las Escuelas por el orden de un Alfabeto Racional. Por Don Vicente. Naharro, Maestro Real y del Colegio de esta corte. Revisor de firmas por el Supremo Consejo, etc. Madrid, Imprenta que fué de Fuentenebro. 1824.

-1818: Memoria premiada por la Real Suprema Junta general de Caridad. Madrid, Imprenta Real, 1818.

-1818: Descripción de los juegos de la infancia, los más propios á desenvolver sus facultades fisicas y morales y para servir de abecedario gimnástico. Por D. Vicente Naharro. Con licencia. Madrid, Imprenta que fué de Fuentenebro. Año de 1818.

-1820: Arte de enseñar á escribir cursivo y liberal, inventado por D. Vicente Naharro, Madrid, Imprenta de Vega y Compañia, 1820.

-1821: Nuevo método de enseñar la arismética por los principios del sistema decimal. Obra destinada al uso de maestros y discípulos de las escuelas de primeras letras. Por Don Vicente Naharro. Madrid, Imprenta que fué de Fuentenebro. Año de 1821.

\section{La justificación del método: el prólogo del Nuevo Arte}

El método que propone Naharro está desarrollado en el prólogo de su Nuevo Arte. Se trata de un plan racional y sistemático, que solo se puede entender a partir de un concepto muy digno y profesional del oficio de maestro $(1824,24)$ :

Los profesores serian como yo digo, si hubiese una cátedra ó escuela normal, donde se enseñase la ciencia de la educacion, como se enseña la filosofia, la medicina, \&. si de consiguiente estuviesen bien dotados, y tuviesen en su vejez una honrosa jubilacion. 
El problema, para nuestro autor, radicaba precisamente en que la enseñanza de la lectura y escritura en España había adolecido hasta ese momento de improvisación y falta de profesionalidad. Por eso, él defiende con orgullo un proyecto racional, y lo presenta como el método más eficaz de su tiempo para la enseñanza de la lectura 5 . Según el autor, con este método se lograba una síntesis superadora de "los tres sistemas en que estaba dividida la opinion de los profesores, á saber en el deletréo, el silabéo, y el de enseñar por los sonidos" $(1824,15)$.

Naharro dedica varias páginas del prólogo a explicar los obstáculos que hubo de superar para lograr la aceptación de una gran parte de los maestros de primera enseñanza. En primer lugar, recuerda, escribió un silabario, al que añadió, en un primer momento, el Método de enseñar el conocimiento de las letras, escrito por José de Anduaga, y completó el conjunto con un libro de lectura, el Libro segundo de los niños, que sustituía al Catón cristiano. Todo ello fue impreso por la Imprenta Real.

Pero cuando el maestro ofreció al Colegio de primeras letras una versión mejorada para dar a imprenta a costa del propio Colegio, los patrocinadores desvirtuaron el silabario y el libro de lectura, lo cual obligó al autor a formar por sí solo "los libros elementales de la enseñanza de leer, aunque no sirviesen sino para el uso de mi escuela" $(1824,18)$. Antes de publicarlo, redactó su ensayo titulado Recopilación de los varios métodos inventados para facilitar la enseñanza de leer, con el fin de fundamentar su silabario racionalmente y con la autoridad de "hombres todos literatos y filósofos". Anejo a este ensayo y también como obra aparte, publicó una versión muy mejorada del silabario, a la que añadió después su propio método práctico de enseñar a leer, distinto del de Anduaga. Además, presentó el silabario acompañado de grandes carteles con caracteres gruesos que debían ser colgados en las aulas (cf. 1824, 20).

El autor no oculta su satisfacción cuando recuerda con cuánta aceptación fue recibido su plan. De hecho, se explaya detallando las diversas maneras en que los maestros utilizaban los carteles y cómo se ve obligado a redactar el Nuevo Arte precisamente para explicar su óptima aplicación en las aulas (1824, 22-3):

Quedaba aún otro vacío que llenar, después de vencer estos inconvenientes como dirémos; porque los carteles, aunque guardaban el mismo órden del silabario en la colocacion de sus números, no habia un tratado, ni un arte, que explicase su mecanismo, en el que los maestros, por convencimiento de la razon, pudiesen enseñar, ni ordenar la enseñanza con aquella seguridad tan necesaria en un nuevo sistema; de manera, que el plan estaba incompleto y defectuoso, como que se iba haciendo por grados segun la experiencia lo iba enseñando; pero ahora creo que ha recibido toda la perfeccion de que es suceptible: y esto es lo que voy á presentar á los profesores con

5 Se cuida Naharro de decir que es el primero. De hecho, ya existían en el ámbito hispanohablante silabarios que sustituían a las viejas cartillas alfabéticas, como los que citan Infantes y Martínez Pereira (2003, 20) y Castañeda (2004, 41), que no tuvieron tanta repercusión: El Silabario teórico-práctico, de Juan Antonio González Valdés y el Silabario metódico, ortológico y ortográfico, de Pedro de Coca y Bermúdez. Otros autores, como Mariano Nifo, defendían el método ya a mediados del XVIII (cf. García Hurtado, 2004, 22). 
aquel órden y escrupulosidad que me ha enseñado el estudio y la razon, dando tambien nueva forma al juego de los carteles, mucho mas completa, mas cómoda y de mayor economía.

Después de recordar estos avatares, Naharro se centra ya en la justificación del método. Según el autor, su éxito se explica por la sencillez, pues "es el método de la naturaleza, el mas fundado en razon y buena lógica", ya que se enseña "por los órganos de la voz (...) del mismo modo que se les enseña a leer" (1824, 26-27). Esto es, el maestro aragonés propone un sistema fonético (ahora veremos que a través de las sílabas), frente a la enseñanza alfabética que provoca el vicio del deletreo. "El deletreo ha tenido orígen en la enseñanza de escribir; porque como allí es preciso hacer letras para formar las palabras: á proporcion que la pluma las va formando, el niño las va nombrando" (1824, 33).

Pero para enseñar a leer no es necesario el nombre de las letras, sino su sonido; por ende, leer no exige saber el alfabeto, ni recitarlo por orden. El razonamiento de Naharro es impecable: "la voz produce sonidos y no letras, ¿por qué no se ha de enseñar á leer por sonidos?" (1824, 34). Las consonantes "no se pueden pronunciar solas sino con una de las vocales sea la que quiera; porque tanto importa que á este carácter $B$ se le nombre $b e$, como que se le llame $b a$, porque el sonido no puede resultar sin una letra sonante o vocal" $(1824,31)$.

Naharro opta, pues, por un método fonético, pero se desmarca de otros que empezaron a aplicarse también en algunas escuelas, como la de Pestalozzi (cf. 1824, 32). Es sabido que durante unos pocos años a principios del XIX fueron aplicados en Madrid y en otras ciudades españolas ${ }^{6}$ el audaz modelo de este maestro suizo que, para la enseñanza de la lectura, comenzaba mostrando los sonidos de la lengua. Según Vicente Naharro (1824, 32-33), el método de Pestalozzi no es válido porque muchos sonidos consonantes requieren, para su ejecución, de una vocal. Por eso, nuestro maestro defiende un método fonético, pero silábico a la vez.

Basta lo dicho hasta el momento para hacerse idea de las dificultades que tuvo que vencer Naharro por neutralizar una inercia de siglos y la oposición de muchos "maestros rancios", como él mismo los califica $(1824,29)$. El prólogo termina con un reto: el maestro aragonés, confiado en que no presenta un modelo teórico, sino un

6 "Tras el éxito de la Escuela pestalozziana creada en 1805 (en la calle Ancha de San Bernardo), el 4 de noviembre de 1806, onomástica del Rey Carlos IV, se inauguró el Real Instituto Militar Pestalozziano (en la calle del Pez), con la doble misión de educar a los cadetes de la Casa de Pajes según el método natural de Pestalozzi, a la vez que de formar a maestros en ejercicio en la metodología del pedagogo suizo. El número de alumnos en este Instituto fue superior al centenar. Tanto el padrino del Instituto, Godoy, como la misma familia real promovieron activamente este ensayo, y su repercusión fue tan grande como para que Goya y su discípulo Agustín Estévez dejaran grabados y pinturas o para que se hicieran publicaciones sobre el método. Los avatares políticos entre 1807 y 1808 dieron al traste con el Instituto" (Ruiz Berrio, 2004, 133). 
método probado, desafía a cualquiera a que, por otro sistema, enseñe a leer mejor y en menos tiempo. Pasamos ahora a analizar este método.

\section{Análisis de la obra ${ }^{7}$}

Naharro va explicando a lo largo de su libro los presupuestos sobre los que se asienta el método racional para la enseñanza de la lectura. Nosotros los sintetizamos en estas tres ideas, que desarrollaremos a continuación: la escritura tiene que reflejar los sonidos de la lengua; existe un orden natural en el aprendizaje; y la dinámica de las clases debe responder a un plan sistemático, bien meditado de antemano.

En primer lugar, la escritura tiene que ser reflejo de los sonidos. Este es un presupuesto basilar, que el autor formula expresamente (p. 67):

Todo el mundo conviene, en que el fin y el deber de la escritura, es representar los sonidos del discurso, lo mas fiel y exactamente que sea posible: Esto es lo que la distingue de las pinturas geroglíficas, y simbólicas, y lo que constituye su prodigiosa utilidad

Por ello, los maestros han de conocer bien las cualidades vocales o características de los sonidos: voz, duración, tono y timbre, los "elementos físicos ó materiales de la palabra" (p. 58). El autor no incluye entre estas cualidades la intensidad, pero sí la voz, como aquello que distingue un sonido de otro ${ }^{8}$.

Naharro insiste en la necesidad de minimizar el perjuicio que ocasiona el hecho de que no exista transparencia entre código escrito y lengua oral, y critica la inercia que, durante siglos, ha llevado a seguir el alfabeto para enseñar a leer, habida cuenta de que los alfabetos se han formado de modo caprichoso y adolecen de muchas incoherencias. Por ejemplo, el nombre de las letras: ¿por qué ache o ge? (cf. pp. 59-60); el propio orden alfabético, en el que se mezclan vocales y consonantes, se distancian las vocales o van seguidas consonantes que se articulan de maneras muy diferentes (cf. p. 61); o, finalmente, la falta de correspondencia entre grafías y sonidos (p. 63):

Unas veces falta un carácter, y se reunen dos para espresar una sola articulacion; otras veces un mismo carácter tiene sucesivamente muchos valores: algunas veces una articulacion no tiene signo que la esprese, y otras veces un mismo signo sirve para tres ó cuatro articulaciones diferentes

Afirma, en suma, el maestro aragonés que los alfabetos son "imperfectas taquigrafías" y "no merecen el nombre de escritura" (p. 64), por lo que se propone emprender una reforma global que dirigiera la Academia y que implicara a todos los estamentos de la sociedad. Naharro reconoce que esta reforma habría de implantarse de

${ }^{7}$ Por abreviar, en este epígrafe las referencias de páginas en las que no se indica año han sido extraídas de la edición de 1824 del Nuevo Arte.

${ }^{8}$ El maestro no deja claro en qué se diferencia la voz de la "articulación", a la que se refiere después: "La articulacion es la diversa modificacion instantánea, principio del sonido, ó como el sonido se empieza á formar" (p. 56). 
modo paulatino y él mismo sugiere que podría empezar por unificar en la $k$ el valor de las grafías con sonido velar $q, c$ y $k$ (p. 69).

Pero no se queda el maestro en una consideración de tipo racional. El sentido de fondo, el motivo último al que apela es la psicología infantil. A este respecto, vale la pena citar un fragmento de la introducción de su obra Recopilación de los varios métodos para enseñar a leer (1801, 5-6):

Un solo exemplo bastará para hacer conocer los inconvenientes de estas inadvertencias.

Se presenta á leer á un niño la palabra guerra: él pronuncia, por el órden de su maestro g-u-e-r-r-a, ve aquí seis sonidos muy llenos seguramente, en los quales su pequeña comprehension va naturalmente á encontrar una idea qualquiera de analogía; pero quál es su sorpresa quando oye á su maestro convertir por solo su poder estas tres vocales y tres consonantes en estos dos sonidos gue-rra.

¿No es un absurdo el hacer digerir á los niños esta inconseqüencia en la edad en que tanto importa cultivar en ellos el espíritu de analogía? ¡qué perspectiva de un trabajo ingrato y desagradable desde el primer paso que dan en la carrera de la instruccion!

Continúa Naharro argumentando que el rechazo de muchos niños hacia la instrucción se debe a los castigos que les son infligidos por la dificultad que acarrea un aprendizaje tan penoso. Del texto además se colige que para el autor una articulación completa es la constituida por cada una de las sílabas, ni siquiera por cada uno de los fonemas. Así, en el ejemplo que ofrece, en guerra hay dos "sonidos", no seis (tantos como letras) ni tampoco cuatro (tantos como lo que hoy llamamos fonemas).

Hay un orden natural en el aprendizaje de los sonidos. El autor no afirma expresamente que exista un orden de adquisición del lenguaje oral, pero se infiere de algunos de sus comentarios: la voz que primero emiten los niños en el parto o el sonido labial del balbuceo. No obstante, sí subraya que hay pronunciaciones más simples y más complejas y que el orden de la enseñanza debe respetar ese grado natural. En consecuencia, Naharro incide en la idea de que las tablas deben respetar un orden preciso, que no será el mismo en todos los idiomas. Para la lengua española él propone el siguiente:

a) Sonidos vocales.

b) Sonidos consonantes.

Labiales.

Dentales.

Palatinos.

Guturales. 
Por otro lado, las consonantes requieren para su articulación de, al menos, un sonido oral puro o vocal. Es decir, las consonantes solo se pueden enseñar por sílabas $\mathrm{y}$, en este aspecto, también conviene ajustarse a una progresión de menor a mayor grado de dificultad articulatoria. La secuencia para cada grupo de consonantes es siempre la misma: usando términos actuales, primero se deben enseñar las sílabas directas abiertas (CV); luego, las inversas (VC); a continuación, las trabadas (CVC); después, los sínfones en sílaba abierta (CCV) y trabada (CCVC); finalmente, se termina con los diptongos (VV, CVV, CVVC, CCVV, CCVVC) y los triptongos (VVV).

Resulta interesante la idea, formulada explícitamente, de que en el encabezamiento de cada tabla no figure el nombre de la consonante, sino su sonido apoyado en la vocal -e (cf. p. 87). De hecho, Naharro postula que a la consonante se la nombre de este modo y, así por ejemplo, que la $g$ sea "gue", no "ge", y que el maestro enseñe "sin decir jamás el nombre de la letra" (p. 149). Solo al final de todo el proceso concede nuestro autor que se muestren en pliegos separados "los alfabetos romano y grifo" (p. 150), pero una vez que los niños ya saben leer y no les puede perjudicar saber el nombre de cada letra.

Este modo de enseñar ha de plasmarse también en una práctica docente racional. Naharro apela a menudo a su experiencia para proponer medidas muy útiles $\mathrm{y}$, sin duda, adelantadas a su tiempo.

En primer lugar, llama la atención el esmero con que describe cómo debe usarse el material didáctico (cf. p. 147): los 50 cartones dobles, pegados con engrudo y colgados de un alambre, que contienen las tablas y que constituye lo que él denomina "atlas" (p. 146), más un solo pliego sinóptico "para que los profesores en las lecciones particulares y los padres de familia, no carezcan de las ventajas de este escelente método" (p. 45). Todo esto sustituiría a la antigua cartilla. Además, como libro de lectura se prescribe su método práctico, en lugar del denostado Catón.

El maestro va salpicando aquí y allá su discurso de interesantes ideas pedagógicas:

Hablar poco y trabajar mucho, son los dos puntos mas esenciales de la enseñanza; porque para entrar en razon con un niño, es necesario ponerse á su nivel, valerse de su modo de entender, de ver, su modo de considerar, de combinar, de juzgar y de racionar. En lugar de salir de la estrecha esfera de sus ideas, es necesario entrar en sí mismo, y sin dejar de ser hombre hecho, volverse niño con él (p. 80).

En consecuencia, es deber del profesor no hacer tediosas las lecciones y evitar los castigos: que las lecciones duren poco y que los descansos ocupen el doble de tiempo que las clases. En este sentido, Naharro recomienda que las sesiones sean breves, pero intensas; incluso que se expliquen dos tablas cada vez, para evitar "la bulla que meten los niños estando ociosos" (p. 148). Se desciende incluso a pormenores como la disposición homogénea de los niños en el aula o se proponen praxis tan razonables como que el maestro se salte el orden de las sílabas en las tablas para evitar que las reciten de memoria (p. 125) o que sean los propios alumnos los que corrijan los errores unos a otros (p. 148). Además, el autor es consciente de que el fin de sus lecciones no es el aprendizaje de las sílabas, sino la lectura y, por ende, aconseja a los profesores 
que, después de cada tabla, formen palabras reales con las sílabas que se han aprendido (ibid.). Y, por eso también, al final de cada clase se incluyen lecciones sumarias dispuestas en nuevas tablas formadas por filas de palabras separadas en sílabas, que puedan servir de prueba o examen (cf. pp. 101-2).

\section{Últimas consideraciones: Naharro, un maestro adelantado a su tiempo}

Pensamos que tal vez no se ha prestado suficiente atención a la concepción que este maestro tiene de la enseñanza elemental, tan diferente de la que prevalecía en su época.

Ya el simple hecho de que recopilara los métodos pedagógicos más avanzados de maestros extranjeros y que mostrara su entusiasmo y admiración por la inclusión en las aulas de juguetes y artilugios educativos (dados, bolas, fichas, naipes, abanicos, etc.), o sus reflexiones acerca de la disposición de los niños en la clase, nos revela bastante del carácter audaz, inquieto e innovador de este maestro aragonés. Naharro (1801, 62-3; 83 ; et passim) se interesa incluso por los materiales -yeso, latón, madera- de que están fabricados dichos artificios, para encontrar el más económico y adecuado a cada situación educativa y a que la instrucción, en esas edades, discurra de manera agradable para los alumnos:

Es necesario hacer agradable á los niños este estudio, no obligándoles á que esten sentados las horas de escuela. Los carteles en sílabas por clases y números repartidos por las paredes de la escuela, contribuirán mucho á que los niños no se fastidien (Naharro, 1801, 147).

Hasta Naharro, en España se enseñaba a leer por el sistema del deletreo, que seguirá vigente aún durante todo el siglo XIX y parte del XX (cf. Viñao 2001, 202). En este sentido, nuestro maestro supone un hito importante en la renovación de los métodos, no solo por generalizar el uso de silabarios -muchos de los cuales se convierten en clásicos: piénsese, por ejemplo, en el de Saturnino Calleja (1876)-, sino por profundizar en una didáctica racional que tuviera en cuenta la correspondencia entre fonema y grafía. En este sentido, métodos onomatopéyicos de lectoescritura tan populares en la España del último tercio del XX como Micho, de la editorial Bruño, se asientan sobre bases muy parecidas.

Por otro lado, Naharro se cuestiona con audacia las inercias pedagógicas que se arrastraban desde la Escolástica, abriendo las puertas a otra manera de plantear la enseñanza en los primeros niveles de instrucción. Así, además de las que ya se han hecho, referidas a la lectura, vamos a apuntar otras dos consideraciones acerca de la mente pedagógica de este maestro.

La primera es la importancia que otorga al ejercicio físico. Algunos estudios singulares han destacado que este maestro es un claro precursor de la materia de Educación Física (cf. Torrebadella, 2011). Ya solo el hecho mismo de que publique un opúsculo de juegos ${ }^{9}$, con sus correspondientes láminas, precedido de un pequeño

${ }^{9}$ Existe alguna edición facsimilar moderna, por ejemplo, la de Maxtor, Valladolid (2001). 
silabario, apunta de manera patente a la importancia de la psicomotricidad en el desarrollo del niño.

En segundo lugar, el concepto que tiene de la escritura. Se ha señalado que uno de los rasgos que caracterizaban a las nuevas formas de enseñanza que afloran en época ilustrada era el de simultanear el aprendizaje de la lectura con el de la escritura. Es esta también una de las preocupaciones de Naharro, plasmadas en su obra de senectud Arte de escribir cursivo y liberal. Tanto se adelanta el maestro a su tiempo que merece el juicio severo de Cotarelo y Mori, quien a principios del siglo XX es incapaz de entender la virtud del método que propone Naharro y que ahora explicaremos brevemente. Sin embargo, tal propuesta es coherente con el pensamiento pedagógico desplegado en el Nuevo Arte, que aquí se ha expuesto, y queda avalado, si confiamos en las palabras del autor, por la experiencia docente de toda una vida en las aulas.

Vicente Naharro es un detractor de la caligrafía, al menos como se entendía en la época y hasta hace relativamente poco tiempo en la escuela española. Para el maestro aragonés, tiene más importancia la rapidez y desenvoltura de los trazos que la obsesión por copiar modelos: "En esto á nadie perjudico sino á los interesados en vender sus muestras" (cit. en Cotarelo, 1916, 107). Su idea de escritura se opone, por tanto, a la del canon caligráfico y se ajusta completamente a un concepto pragmático de escuela infantil en la que:

1) Los niños aprenden con más soltura porque no escriben agobiados por no cometer borrones y ser castigados, ya que el soporte utilizado no es papel y tinta indeleble, sino hojas de lata sobre las que la tintura no se fija.

2) No se coarta el trazo con una ingente cantidad de reglas de pautado: al contrario, el niño desarrolla su propia letra.

3) Se busca ejercitar el movimiento y una postura correcta, cómoda y práctica.

El método de Naharro va, por tanto, más allá de un sistema para enseñar a escribir. Disponer que los alumnos escriban de pie para no perder tiempo en levantarse y sentarse (es el maestro quien se pasea entre el grupo), o que sean los propios niños los encargados de regar y barrer el aula, porque esto "conducirá infinito á su educación física", dice mucho de su amplitud de miras y de la audacia de sus planteamientos. El sabio maestro cuenta con el error, porque es parte del aprendizaje, como afirma en su Recopilación (1801, 149-50):

Es natural que el niño se equivoque en su composicion, y que por escribir guitarra, por exemplo, escriba gitara; pero esta misma equivocacion puede suministrar al maestro varias reflexiones: puede decirle que esta es la ortografia natural: que nadie escribe esta palabra sin u despues de la $g$, ni con una sola $r$ : de este modo volvereis en provecho su misma falta. (...) Así se empieza á formar el juicio de los niños; y es el modo como se puede $\mathrm{y}$ aun es necesario proceder con ellos en todo para hacerles pasar insensiblemente de las ideas simples á las ideas compuestas.

Cotarelo no comparte esas ideas, tan diferentes a la de la enseñanza tradicional de la escritura: "Considerado como método de escritura absoluto es un verdadero desatino; porque con tal libertad cada uno escribiría como le pareciese y llegaría momento en 
que nadie supiese leer lo que los demás escribiesen" (1916, 109). Por eso, tilda a Naharro de poco ingenioso con el argumento de que ya otros maestros utilizaban las hojas de lata como soporte, critica con el testimonio de ilustres calígrafos que se oponga la rapidez a la elegancia o tacha a nuestro maestro de incoherente por dar demasiada libertad y pretender a la vez que las clases discurran con orden (Cotarelo, 1916, 109):

Afortunadamente, el mismo Naharro pone luego algún coto á este libertinaje, pues recomienda que después que los niños escriban diez ó doce planas en la lata, se les haga escribir "una planita en papel con algún caído y buena formación; porque también sucede que por este medio se sueltan tanto los niños que disparatan y no forman muy bien, y es necesario tirarles la rienda de cuando en cuando, haciéndoles escribir papel".

Sin embargo, todo apunta a que Naharro sabía bien lo que se hacía y que era consciente de las incomprensiones de que sería objeto:

Griten, muerdan, murmuren; que yo les haré á los profesores y á los niños un beneficio contra todo el torrente de los envidiosos, y desterraré con éste todos los métodos de la Europa (cit. en Cotarelo, 1916, 107).

A las críticas, el maestro aragonés opone el testimonio de una vida entregada a la educación de los niños. Son los progresos de sus alumnos lo que le concede toda su autoridad: "He logrado lo que deseaba, pues habré hecho un servicio á mi patria, y un beneficio á la infancia, que es á todo lo que yo podia aspirar" (Naharro, 1801, 160).

$$
* * *
$$

La profundización historiográfica en la figura y la obra de Vicente Naharro nos ha permitido descubrir aspectos interesantes de una mente pedagógica muy adelantada a su tiempo. No es pretencioso, a tenor de lo expuesto en este breve artículo, que se reivindique para este maestro un puesto de honor en la historia de la Educación en España.

\section{Referencias bibliográficas}

CALERO VAQUERA, M. L. (2007). La Cartilla para enseñar a leer en romance (1564) de Juan Robles, En CANO LÓPEZ, P. (coord.), Actas del Congreso de Lingüística General. Santiago de Compostela, 3-7 de mayo de 2004 (vol. 3) (27452768). Madrid: Arco Libros.

CASTAÑEDA, C. (2004). Libros para la enseñanza de la lectura en la Nueva España, siglos XVIII y XIX: cartillas, silabarios, catones y catecismos, En CASTAÑEDA, C. y GALVÁn, L. E. y MARTínez MOCTEZUMA, L. (coords.), Lecturas y lectores en la historia de México (35-66). México: CIESAS, El Colegio de Michoacán y Universidad Autónoma del Estado de Morelos.

COTARELO Y MORI, E. (1916). Diccionario biográfico y bibliográfico de calígrafos españoles (vol. 2). Madrid: Imprenta de la Revista de Archivos, Bibliotecas y Museos. 
ESPARZA, M. Á. (Dir.), ÁlvareZ, A., BATTANER, E., CAlVO, V. Y RODRÍGUEZ, S. (2008). Bibliografia temática de historiografia lingüística española. Fuentes secundarias. Hamburg: Buske.

ESPARZA, M. Á. Y NIEDEREHE, H.-J. (dirs.) ÁlVAREZ, A., BATTANER, E., CALVO, V. HAOUET, L. Y RODRÍGUEZ, S. (cols.) (2012). Bibliografía cronológica de la lingüística, la gramática y la lexicografia del español (BICRES). Desde el año 1801 hasta el año 1860. Amsterdam/Philadelphia: John Benjamins.

GARCÍA HURTADO, M. R. (2004). Reflexiones sobre algunos textos destinados a enseñar a leer y escribir en España entre 1700 y 1780. Obradoiro de historia moderna, 13, 7-38.

INFANTES, V. (1998). De las primeras letras. Cartillas españolas para enseñar a leer de los siglos XV y XVI. Salamanca: Ediciones Universidad.

- Y MARTÍNEZ PEREIRA, A. (2003). De las primeras letras. Cartillas españolas para enseñar a leer de los siglos XVII y XVIII. Salamanca: Ediciones Universidad.

LEBRERO, M. P. Y LEBRERO, M. T. (1999 ${ }^{4}$. Cómo y cuando enseñar a leer y a escribir. Madrid: Síntesis.

NAHARRO, V. (1802). Recopilación de los varios métodos inventados para facilitar la enseñanza de leer. Madrid: [En la oficina de D...].

- (1821). Arte de enseñar á escribir cursivo y liberal. Madrid: Imprenta de Vega y Compañía.

- (1824). El Nuevo Arte de enseñar a leer a los niños de las Escuelas por el orden de un Alfabeto Racional. Madrid: Imprenta que fue de Fuentenebro.

NIEDEREHE, H.-J. (2005). Bibliografía cronológica de la lingüistica, la gramática y la lexicografía del español (BICRES). Desde el año 1701 hasta el año 1800. Amsterdam/Philadelphia: John Benjamins.

RUIZ BERRIO, J. (1997). La enseñanza de la lectura de la Ilustración al liberalismo, En Escolano, A. (Dir.), Historia ilustrada del libro escolar en España. Del Antiguo Régimen a la Segunda República (193-227). Madrid: Fundación Germán Sánchez Ruipérez.

- (2004). Maestros y escuelas de Madrid en el Antiguo Régimen. Cuadernos de Historia Moderna. Anejos, III, 113-135.

- (2009). Los manuales y los objetos escolares como instrumentos de reforma educativa en el reinado de Carlos IV, en LORENZO ÁlVAREZ, Elena de (coord.), La época de Carlos IV, (1788-1808): Actas del IV Congreso Internacional de la Sociedad Española de Estudios del Siglo XVIII (1021-1032). Oviedo: Trea.

TORRE REVELLO, J. (1960). Las cartillas para enseñar a leer a los niños en América española. Thesaurus: Boletín del Instituto Caro y Cuervo, 15 (1-3), 214-234. 
TORREBADELLA FLIX, X. (2011). Vicente Naharro y los juegos corporales en la educación física española de la primera mitad del siglo XIX. Ágora para la Educación Física y el Deporte, 13 (2), 165-182.

VIÑAO FRAGO, A. (1997). Aprender a leer en el Antiguo Régimen: cartillas, silabarios y catones, en ESCOLANO, A. (Dir.), Historia ilustrada del libro escolar en España. Del Antiguo Régimen a la Segunda República (149-191). Madrid: Fundación Germán Sánchez Ruipérez.

- (1998). Alfabetización e ilustración, diez años después (de las evidencias directas a las indirectas). Bulletin Hispanique, 100 (2), 255-269.

- (2001). Los discursos sobre la lectura en la España del siglo XIX y primeros años del XX, En MARTíNeZ MOCTEZUMA, L. (coord.), La infancia y la cultura escrita (191-242). México DF/Buenos Aires: Siglo Veintiuno.

- (2002a). La enseñanza de la lectura y la escritura: análisis socio-histórico. Anales de documentación, 5, 345-359.

- (2002b). Towards a Typology of the Primers for Learning to Read (Spain, c. 14961825). Paedagogica Historica, 38 (1), 73-90.

\section{Correspondencia con el autor}

Vicente CALVO FERNÁNDEZ

Universidad Rey Juan Carlos - Campus de Fuenlabrada

Edif. Departamental III, Desp. 007

$\mathrm{C}^{\mathrm{o}}$ del Molino $\mathrm{s} / \mathrm{n}$

28943 FUENLABRADA (Madrid)

Correo-e: vicente.calvo.fernandez@urjc.es 